PROCEEDINGS OF THE

AMERICAN MATHEMATICAL SOCIETY

Volume 135, Number 2, February 2007, Pages 329-335

S 0002-9939(06)08513-3

Article electronically published on August 1, 2006

\title{
COMPLETE INTERSECTIONS IN TORIC IDEALS
}

\author{
EDUARDO CATTANI, RAYMOND CURRAN, AND ALICIA DICKENSTEIN
}

(Communicated by Michael Stillman)

\begin{abstract}
We present examples that show that in dimension higher than one or codimension higher than two, there exist toric ideals $I_{A}$ such that no binomial ideal contained in $I_{A}$ and of the same dimension is a complete intersection. This result has important implications in sparse elimination theory and in the study of the Horn system of partial differential equations.
\end{abstract}

\section{INTRODUCTION}

Given a configuration $A=\left\{a_{1}, \ldots, a_{n}\right\} \subset \mathbb{Z}^{m}$ of integral points generating $\mathbb{Z}^{m}$, the (toric) ideal $I_{A} \subset \mathbb{C}\left[x_{1}, \ldots, x_{n}\right]$ is generated by all binomials

$$
x^{u}-x^{v},
$$

whose exponents $u, v \in \mathbb{N}^{n}$ satisfy $A \cdot u=A \cdot v$. Here we are also denoting by $A$ the $m \times n$ matrix whose $j$-th column is $a_{j}$. Note that $I_{A}$ is weighted-homogeneous for every weight $w$ in the row-span of $A$. We shall assume that $(1, \ldots, 1)$ is in the row-span of $A$ and, consequently, that $I_{A}$ is a (standard) homogeneous ideal.

The configuration $A$ and its associated toric ideal $I_{A}$ are the central characters in several areas of very active research in commutative algebra, algebraic geometry, and differential algebra. The associated projective variety $X_{A}$ has a natural action of the algebraic torus $\left(\mathbb{C}^{*}\right)^{m}$ making it into a projective toric variety of dimension $d:=m-1$. We call $d$ the dimension of $A$.

Let $\mathcal{L}_{A} \subset \mathbb{R}^{n}$ be the lattice

$$
\mathcal{L}_{A}=\left\{v \in \mathbb{Z}^{n}: A \cdot v=0\right\}
$$

and let $r:=n-m$ be the codimension of $A$. Let $B=\left\{v_{1}, \ldots, v_{r}\right\} \subset \mathcal{L}_{A}$ be a maximal set of vectors linearly independent over $\mathbb{Q}$. We also denote by $B$ the $n \times r$ matrix whose $j$-th column is $v_{j}$ and consider the binomial ideal $J_{B}$ generated by the binomials $x^{v_{j}^{+}}-x^{v_{j}^{-}}$, where $v_{j}=v_{j}^{+}-v_{j}^{+}$is the decomposition in positive and negative components.

It has been proven in $[8]$ that $J_{B} \cdot \mathbb{C}\left[x_{1}^{ \pm 1}, \ldots, x_{n}^{ \pm 1}\right]$ is always a complete intersection in the Laurent polynomial ring, and that it coincides with $I_{A} \cdot \mathbb{C}\left[x_{1}^{ \pm 1}, \ldots, x_{n}^{ \pm 1}\right]$ if and only if the greatest common divisor $g$ of the maximal minors of $B$ satisfies $g=1$. This positive integer $g$ is precisely the index with respect to $\mathcal{L}_{A}$ of the lattice spanned by $B$. We will refer to $J_{B}$ as a basis ideal. Following [14 we will reserve

Received by the editors January 11, 2005 and, in revised form, August 18, 2005.

2000 Mathematics Subject Classification. Primary 14M10; Secondary 14M25, 13C40.

The first author was partially supported by NSF Grant DMS-0099707.

The third author was partially supported by UBACYT X042, Argentina.

(C)2006 American Mathematical Society 
the term lattice basis ideal for the case when $B$ is a $\mathbb{Z}$-basis of $\mathcal{L}_{A}$. Clearly $J_{B} \subset I_{A}$ and, in general, this containment is proper.

In this note we study a question that arises naturally in the study of $A$-discriminants [7] and of Horn systems of differential equations [6]: does every toric ideal contain a complete intersection basis ideal (i.e., a complete intersection binomial ideal of the same dimension)? This is indeed the case if $X_{A}$ is a monomial curve $(d=1)$ or in the codimension two case $(r=2)$. The purpose of this note is to show that in any dimension higher than one and any codimension higher than two, there exist toric ideals $I_{A}$ such that no basis ideal contained in $I_{A}$ is a complete intersection.

We recall that the dual variety $X_{A}^{*}$ of $X_{A}$ is the Zarisky closure of the locus of hyperplanes tangent to $X_{A}$ at a smooth point. When $X_{A}^{*}$ is a hypersurface, its defining equation is the $A$-discriminant. This notion, introduced by Gel'fand, Kapranov, and Zelevinsky [12, generalizes the classical notion of the discriminant of a univariate polynomial. Dickenstein and Sturmfels [7] have shown how to compute $A$-discriminants for codimension two (i.e., $n-m=2$ ) configurations. A key ingredient of this work is the fact that every basis ideal in a codimension two toric ideal is a complete intersection. Our results show that a different approach is needed to describe $A$-discriminants in higher codimensions.

The work of Gel'fand et al. on sparse elimination was a step toward the study of $A$-hypergeometric (or GKZ) systems. Consider the "quantized" version of the ideal $I_{A}$, that is, the left ideal $H_{A}(\beta)$ in the Weyl algebra

$$
D_{n}=\mathbb{C}\left\langle x_{1}, \ldots, x_{n}, \partial_{1}, \ldots, \partial_{n}\right\rangle
$$

generated by the toric operators $\partial^{u}-\partial^{v}, A \cdot u=A \cdot v$ together with the Euler operators associated with the $\left(\mathbb{C}^{*}\right)^{m}$ action:

$$
a_{j 1} x_{1} \partial_{1}+\cdots+a_{j n} x_{n} \partial_{n}-\beta_{j},
$$

where $\beta=\left(\beta_{1}, \ldots, \beta_{m}\right) \in \mathbb{C}^{m}$. An $A$-hypergeometric function of degree $\beta$ is a locally defined (multivalued) holomorphic function $\mathbb{C}^{n}$ annihilated by $H_{A}(\beta)$. This notion of hypergeometric functions encompasses most of the classical univariate and multivariate hypergeometric functions. GKZ systems are holonomic for all choices of parameters $\beta$, and so in particular, the corresponding spaces of $A$-hypergeometric functions are finite dimensional. Another classical multivariable generalization of hypergeometric differential equations is given by the Horn systems, which are closely related to the GKZ systems. A Horn system consists of the Euler operators and only those toric operators coming from a basis ideal $J_{B}$ contained in $I_{A}$. Dickenstein, Matusevich, and Sadykov [6] have shown that, in codimension two, the behavior of an $A$-hypergeometric system and that of any of its associated Horn systems is not very different. This relies on the fact that such basis ideals are complete intersections. However, if the basis ideal is not a complete intersection, the Horn system has an infinite dimensional local solution space for all choices of parameters and, thus, is never holonomic. Our examples show that in the general multivariate case, the $A$-hypergeometric and Horn systems have essentially distinct behavior.

\section{Preliminaries}

The study of binomial ideals is intimately connected with the study of (affine) semigroup algebras. It is from this perspective, and beginning with the work of Herzog [13] and Delorme [5, that the question of classifying complete intersection 
binomial ideals has been extensively studied by many authors [1, 2, 3, 9, 10, 11, 14, 15, 16, 17, 18, 20, 21, 22. A combinatorial characterization of these ideals is given in [11] in terms of a choice of $B$ and the notion of mixed matrices; that is, matrices such that every column contains a strictly positive and a strictly negative entry. Note that since the columns of the matrix $B$ add up to zero, $B$ is automatically mixed. The following result follows from [8, Theorem 2.1] and [11, Theorem 2.3] (see also [17, Theorem 2.7]).

Theorem 2.1. The ideal $J_{B}$ is a complete intersection if and only if for every mixed $n^{\prime} \times r^{\prime}$-submatrix $B^{\prime} \subset B$ we have $n^{\prime} \geq r^{\prime}$.

Since a mixed submatrix must contain at least two rows it follows that:

Corollary 2.2. If $r \leq 2$, every basis ideal $J_{B}$ is a complete intersection.

It is also easy to prove that if $m=2$, i.e., for $X_{A}$ a monomial projective curve, there exists $B$ such that $J_{B}$ is a complete intersection. Indeed, let

$$
A=\left(\begin{array}{cccc}
1 & 1 & \cdots & 1 \\
a_{1} & a_{2} & \cdots & a_{n}
\end{array}\right)
$$

where $a_{1} \leq \cdots \leq a_{n}$ are coprime. Performing a row operation that does not change $I_{A}$, we may assume without loss of generality that $a_{1}=0$, and therefore all $a_{j} \geq 0$. Consider now the following choice of $B$ :

$$
B=\left(\begin{array}{cccc}
a_{3}-a_{2} & a_{4}-a_{3} & \cdots & a_{n}-a_{n-1} \\
-a_{3} & 0 & \cdots & 0 \\
a_{2} & -a_{4} & \cdots & 0 \\
0 & a_{3} & \ddots & 0 \\
\vdots & \vdots & \ddots & -a_{n} \\
0 & 0 & \cdots & a_{n-1}
\end{array}\right) .
$$

Since $a_{i+1}-a_{i} \geq 0$, it follows that every mixed submatrix of $B$ must contain more rows than columns and therefore $J_{B}$ is a complete intersection.

Note that already in the simplest case of the twisted cubic; i.e., the curve $X_{A}$ associated with

$$
A=\left(\begin{array}{llll}
1 & 1 & 1 & 1 \\
0 & 1 & 2 & 3
\end{array}\right)
$$

the toric ideal $I_{A}$ is not a complete intersection, but it does contain a complete intersection basis ideal.

Given a vector $v \in \mathbb{Z}^{n}$, we define the support of $v$ :

$$
\operatorname{supp}(v):=\left\{i \in\{1, \ldots, n\}: v_{i} \neq 0\right\} .
$$

Similarly we set $\operatorname{supp}^{+}(v):=\left\{i: v_{i}>0\right\}, \operatorname{supp}^{-}(v):=\left\{i: v_{i}<0\right\}$. If $u, v \in \mathbb{Z}^{n}$, we say that $u$ is conformal to $v$ if $\operatorname{supp}^{+}(u) \subset \operatorname{supp}^{+}(v)$ and $\operatorname{supp}^{-}(u) \subset \operatorname{supp}^{-}(v)$.

Given a configuration $A=\left\{a_{1}, \ldots, a_{n}\right\} \subset \mathbb{Z}^{m}$, a vector $v \in \mathcal{L}_{A}$ is called a circuit if its support is minimal among all elements in $\mathcal{L}_{A}$ relative to inclusion. As shown in the proof of [19, Lemma 4.9], if $u$ is a circuit in $\mathcal{L}_{A}$ of maximal dimension $m$ with $\operatorname{supp}(u)=\left\{i_{1}, \ldots, i_{m+1}\right\}$, then up to a multiple:

$$
u=\sum_{j=1}^{m+1}(-1)^{j} \operatorname{det}\left(a_{i_{i}}, \ldots, a_{i_{j-1}}, a_{i_{j+1}}, \ldots, a_{i_{m+1}}\right) e_{i_{j}} .
$$

The following is Lemma 4.10 in [19]. 
Lemma 2.3. Every vector $v \in \mathcal{L}_{A}$ may be written as a non-negative rational linear combination of $n-m$ circuits each of which is conformal to $v$.

Definition 2.4. Given a configuration $A=\left\{a_{1}, \ldots, a_{n}\right\} \subset \mathbb{Z}^{m}$, we say that a basis ideal $J_{B}$ is generated by circuits if and only if each of the column vectors of $B$ is a circuit in $\mathcal{L}_{A}$.

Proposition 2.5. A toric ideal $I_{A}$ contains a complete intersection basis ideal if and only if it contains a complete intersection basis ideal generated by circuits.

Proof. Let $\left\{v_{1}, \ldots, v_{r}\right\} \subset \mathcal{L}_{A}$ be a $\mathbb{Q}$-linearly independent set defining a complete intersection basis ideal. Let $i \in\{1, \ldots, r\}$ be the smallest index such that $v_{i}$ is not a circuit. Using Lemma 2.3 write $v_{i}$ as a non-negative rational combination of circuits $w_{1}^{i}, \ldots, w_{r}^{i}$ :

$$
v_{i}=q_{1} w_{1}^{i}+\cdots+q_{r} w_{r}^{i} .
$$

Clearly, some $w_{j}^{i}$ must be linearly independent from $\left\{v_{k}, k \neq i\right\}$, hence we may replace $v_{i}$ by the circuit $w_{j}^{i}$ to obtain a new $\mathbb{Q}$-linearly independent set. Continuing in this manner we obtain a linearly independent set $\left\{w_{1}, \ldots, w_{r}\right\}$ consisting of circuits and such that $w_{i}$ is conformal to $v_{i}$.

Let $B$ (respectively $C$ ) denote the matrix whose columns are the vectors $v_{i}$ (respectively $w_{i}$ ). Since $w_{i}$ is conformal to $v_{i}$, it follows that if $C^{\prime}$ is a mixed submatrix of $C$, then the corresponding submatrix $B^{\prime}$ of $B$ is also mixed. Hence, by Theorem 2.1] if $J_{B}$ is a complete intersection, so is $J_{C}$.

\section{TORIC IDEALS OF Dimension AT LEAST TWO}

In this section we will exhibit examples of configurations in any dimension $d=m-1 \geq 2$, which do not contain any complete intersection basis ideal. By assumption we may suppose that $A \subset\{1\} \times \mathbb{Z}^{d}$. By abuse of notation we will identify $A$ with its projection onto $\mathbb{Z}^{d}$.

Theorem 3.1. Let $A$ be the vertex set of a lattice polytope in $\mathbb{R}^{d}$ and $n=|A|$. There exists $N=N(d)$ such that, for $n \geq N, I_{A}$ does not contain any complete intersection basis ideal.

Proof. Let $B \subset \mathbb{Z}^{n \times(n-m)}$ be such that its columns are a $\mathbb{Q}$-basis of the kernel lattice $\mathcal{L}_{A}$. Note that every column of $B$ must contain at least two strictly positive and two strictly negative entries since, otherwise, one of the points in $A$ would be in the convex hull of some of the other points in $A$ and that is impossible by assumption.

Suppose $J_{B}$ is a complete intersection. It then follows from Theorem 2.1 that every $(n-m-1) \times(n-m)$ minor of $B$ must be non-mixed. In other words, for any subset $J \subset\{1, \ldots, n\},|J|=m+1$, there exists a column $v_{i}$ of $B$ such that either the positive support $\operatorname{supp}^{+}\left(v_{i}\right)$ or the negative support $\operatorname{supp}^{-}\left(v_{i}\right)$ is contained in $J$. Clearly, there are $\left(\begin{array}{c}n \\ d+2\end{array}\right)$ index sets $J$ of cardinality $m+1=d+2$.

Now, for a given $v_{i}$, its positive support contains at least two indices and therefore it may be contained in at most $\left(\begin{array}{c}n-2 \\ d\end{array}\right)$ distinct index sets $J$ of cardinality $m+1$. Thus, since $B$ has $n-d-1$ columns and taking into account the positive and negative supports, the condition may be satisfied for at most

$$
2(n-d-1)\left(\begin{array}{c}
n-2 \\
d
\end{array}\right)
$$


index sets $J$. But for $n$ sufficiently large

$$
\left(\begin{array}{c}
n \\
d+2
\end{array}\right)>2(n-d-1)\left(\begin{array}{c}
n-2 \\
d
\end{array}\right)
$$

since the left-hand side is a polyomial in $n$ of degree $d+2$ with positive leading term, while the right-hand side is a polynomial in $n$ of degree $d+1$.

Remark 3.2. In the planar case $d=2$, the inequality (3.1) is satisfied for $n \geq 22$. However, it is clear that the estimates above are very rough and that one should expect Theorem 3.1 to hold for $n$ considerably smaller than 22 .

Example 3.3. Consider a configuration $\left\{a_{1}, \ldots, a_{10}\right\}$ of ten points in $\mathbb{Z}^{2}$ that are the vertices of a polygon. We may assume them to be ordered counterclockwise. Given four indices $1 \leq i<j<k<\ell \leq 10$, there exists a relation

$$
\lambda_{i} a_{i}-\lambda_{j} a_{j}+\lambda_{k} a_{k}-\lambda_{\ell} a_{\ell}=0,
$$

where $\lambda_{i}, \lambda_{j}, \lambda_{k}, \lambda_{\ell}$ are positive integers. Such a relation defines a circuit in the lattice kernel $\mathcal{L}_{A}$. Using the computer algebra system CoCoA [4, we searched for sets of seven such relations satisfying the condition in Theorem 2.1] The following is such an example. We have only indicated the sign of the coefficients since that is all that matters in Theorem 2.1 and, for generic coefficients, the matrix $B$ will be of maximal rank.

$$
B=\left(\begin{array}{ccccccc}
+ & + & + & 0 & + & 0 & 0 \\
- & - & 0 & 0 & 0 & 0 & 0 \\
+ & 0 & 0 & 0 & - & + & 0 \\
- & + & - & 0 & 0 & 0 & 0 \\
0 & 0 & 0 & + & 0 & - & 0 \\
0 & 0 & 0 & - & + & + & + \\
0 & 0 & 0 & + & 0 & 0 & - \\
0 & 0 & + & 0 & - & 0 & + \\
0 & - & - & 0 & 0 & 0 & 0 \\
0 & 0 & 0 & - & 0 & - & -
\end{array}\right) .
$$

We were unable to obtain similar examples with $n=11$. We suspect that Theorem 3.1 holds for polygons in the plane with at least 11 vertices.

\section{TORIC IDEALS OF CODIMENSION AT LEAST THREE}

It follows from Remark 3.2 that Theorem 3.1 furnishes examples of toric ideals that do not contain any complete intersection basis ideal in codimension greater than twenty-one. In this section we will describe a different class of examples that show that in every codimension greater than two there exist toric ideals with that same property.

Let $n=m+r$, and consider a configuration consisting of the vertices of a cyclic polytope (we refer to 23 for other properties of this important class of polytopes):

$$
A=\left(\begin{array}{llll}
1 & 1 & \cdots & 1 \\
t_{1} & t_{2} & \cdots & t_{n} \\
t_{1}^{2} & t_{2}^{2} & \cdots & t_{n}^{2} \\
\vdots & \vdots & & \vdots \\
t_{1}^{m-1} & t_{2}^{m-1} & \cdots & t_{n}^{m-1}
\end{array}\right)
$$


where $0<t_{1}<t_{2}<\cdots<t_{n}$ are integers. For appropriate choices of $t_{1}, \ldots, t_{n}$, the columns of $A \operatorname{span} \mathbb{Z}^{m}$.

Theorem 4.1. Let $r \geq 3$. For $n \geq 2\left(r^{2}-r+1\right)$ the toric ideal $I_{A}$ associated with the matrix (4.1) does not contain any complete intersection basis ideal.

Proof. According to Proposition 2.5, it suffices to show that $I_{A}$ does not contain any complete intersection basis ideal generated by circuits that, up to constant, are given by the expression (2.3). On the other hand, note that all maximal minors of $A$ are non-zero and the determinant

$$
\operatorname{det}\left(a_{i_{i}}, \ldots, a_{i_{j-1}}, a_{i_{j+1}}, \ldots, a_{i_{m+1}}\right)
$$

is strictly positive since it is the Vandermonde determinant for

$$
t_{i_{i}}<\cdots<t_{i_{j-1}}<t_{i_{j+1}}<\cdots<t_{i_{m+1}} .
$$

Therefore, if $v \in \mathcal{L}_{A}$ is a circuit, then it will have exactly $r-1$ zero entries while the remaining entries will alternate in sign.

This means that if $B$ is an $n \times r$ matrix whose columns are a circuit basis of $\mathcal{L}_{A}$, then each column of $B$ contains exactly $r-1$ entries that are zero and $B$ contains a total of $r(r-1)$ zero entries. This implies that if $n \geq 2\left(r^{2}-r+1\right), B$ will have to contain two consecutive rows all of whose entries are non-zero. Let $B^{\prime}$ denote the $2 \times 3$ submatrix consisting of those two rows. Since the signs of the columns are alternating, $B^{\prime}$ is mixed and, by Theorem 2.1, $J_{B}$ is not a complete intersection. In fact, if $B^{\prime}$ consists of the $j$-th and $k$-th rows of $B$, the codimension two ideal $\left\langle x_{j}, x_{k}\right\rangle$ is an associated prime ideal of $J_{B}$.

Remark 4.2. For $r=3$ the lower bound in Theorem 4.1 is 14 . One can show that for $n \leq 11$ every toric ideal $I_{A}$ admits a complete intersection basis ideal. We do not know if there are examples of configurations with $n=12$ or $n=13$ that do not admit any such basis ideals.

\section{ACKNOWLEDGMENTS}

We are grateful to Bernd Sturmfels for many helpful conversations and to John Abbott and Lorenzo Robbiano of the CoCoA group for their programming assistance. We would also like to thank an anonymous referee for very useful comments.

\section{REFERENCES}

[1] M. Barile, M. Morales, and A. Thoma. On simplicial toric varieties which are set-theoretic complete intersections. J. Algebra, 226(2):880-892, 2000. MR.1752767 (2001i:14066)

[2] M. Barile, M. Morales, and A. Thoma. On free complete intersections. In Geometric and combinatorial aspects of commutative algebra (Messina, 1999), volume 217 of Lecture Notes in Pure and Appl. Math., pages 1-9. Dekker, New York, 2001. MR1824213(2002c:13026)

[3] M. Barile, M. Morales, and A. Thoma. Set-theoretic complete intersections on binomials. Proc. Amer. Math. Soc., 130(7):1893-1903, 2002. MR1896020 (2003f:14058)

[4] CoCoATeam. CoCoA: a system for doing Computations in Commutative Algebra. Available at http://cocoa.dima.unige.it.

[5] C. Delorme. Sous-monoïdes d'intersection complète de N. Ann. Sci. École Norm. Sup. (4), 9(1):145-154, 1976. MR0407038 (53:10821)

[6] A. Dickenstein, L. F. Matusevich, and T. Sadykov. Bivariate hypergeometric D-modules. Advances in Mathematics, 196(1):78-123, 2005. MR2159296

[7] A. Dickenstein and B. Sturmfels. Elimination theory in codimension 2. J. Symbolic Comput., 34:119-135, 2002. MR 1930829 (2003h:14073) 
[8] D. Eisenbud and B. Sturmfels. Binomial ideals. Duke Math. J., 84(1):1-45, 1996. MR1394747 (97d:13031)

[9] K. G. Fischer, W. Morris, and J. Shapiro. Affine semigroup rings that are complete intersections. Proc. Amer. Math. Soc., 125(11):3137-3145, 1997. MR1401741 (97m:13026)

[10] K. G. Fischer, W. Morris, and J. Shapiro. Mixed dominating matrices. Linear Algebra Appl., 270:191-214, 1998. MR1484081 (98j:15029)

[11] K. G. Fischer and J. Shapiro. Mixed matrices and binomial ideals. J. Pure Appl. Algebra, 113(1):39-54, 1996. MR1411645 (97h:13008)

[12] I.M. Gel'fand, M.M. Kapranov, and A.V. Zelevinsky. Discriminants, Resultants and Multidimensional Determinants. Birkhäuser, Boston, 1994. MR1264417 (95e:14045)

[13] J. Herzog. Generators and relations of abelian semigroups and semigroup rings. Manuscripta Math., 3:175-193, 1970. MR0269762 (42:4657)

[14] S. Hoşten and J. Shapiro. Primary decomposition of lattice basis ideals. J. Symbolic Comput., 29(4-5):625-639, 2000. Symbolic computation in algebra, analysis, and geometry (Berkeley, CA, 1998). MR:1769658 (2001h:13012)

[15] H. Nakajima. Affine torus embeddings which are complete intersections. Tohoku Math. J. (2), 38(1):85-98, 1986. MR0826766 (87c:14058)

[16] J. C. Rosales and P. A. García-Sánchez. On complete intersection affine semigroups. Comm. Algebra, 23(14):5395-5412, 1995. MR1363611 (96m:14068)

[17] G. Scheja, O. Scheja, and U. Storch. On regular sequences of binomials. Manuscripta Math., 98(1):115-132, 1999. MR1669583 (99k:13017)

[18] R. P. Stanley. Relative invariants of finite groups generated by pseudoreflections. J. Algebra, 49(1):134-148, 1977. MR0460484 (57:477)

[19] B. Sturmfels. Gröbner bases and convex polytopes, volume 8 of University Lecture Series. American Mathematical Society, Providence, RI, 1996. MR.1363949 (97b:13034)

[20] A. Thoma. Construction of set theoretic complete intersections via semigroup gluing. Beiträge Algebra Geom., 41(1):195-198, 2000. MR1745589(2001h:14059)

[21] A. Thoma. On the binomial arithmetical rank. Arch. Math. (Basel), 74(1):22-25, 2000. MR:1728358 (2001a:14023)

[22] K. Watanabe. Invariant subrings which are complete intersections. I. Invariant subrings of finite abelian groups. Nagoya Math. J., 77:89-98, 1980. MR0556310 (82d:13020)

[23] G. M. Ziegler. Lectures on polytopes. Springer, New York, 1995. MR.1311028 (96a:52011)

Department of Mathematics and Statistics, University of Massachusetts, Amherst, MASSACHUSETtS 01003

E-mail address: cattani@math.umass.edu

Department of Mathematics and Statistics, University of Massachusetts, Amherst, Massachusetts 01003

Current address: Department of Mathematical and Computer Sciences, Metropolitan State College of Denver, Denver, Colorado 80202

E-mail address: rcurran@mscd.edu

Departamento de Matematica, FCEyn, Universidad de Buenos Aires, (1428) Buenos Aires, Argentina

E-mail address: alidick@dm.uba.ar 